\title{
Liquid Phase Exfoliation of Rubrene Single Crystals into Nanorods and Nanobelts
}

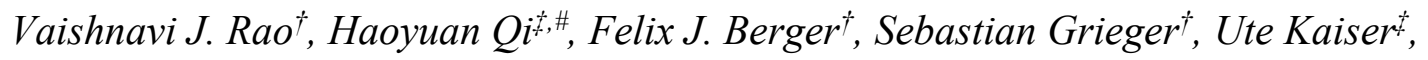
Claudia Backes ${ }^{\dagger} *$ and Jana Zaumseil ${ }^{\dagger} *$

†Institute for Physical Chemistry, Universität Heidelberg, D-69120 Heidelberg, Germany

${ }^{\ddagger}$ Central Facility of Materials Science Electron Microscopy, Universität Ulm, 89081 Ulm, Germany

${ }^{\#}$ Center for Advancing Electronics Dresden \& Faculty of Chemistry and Food Chemistry, Technische Universität Dresden, 01062 Dresden, Germany

\section{Corresponding Authors}

*Email: zaumseil@uni-heidelberg.de

*Email: backes@uni-heidelberg.de 


\section{Table of Contents}

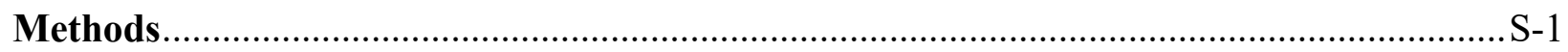

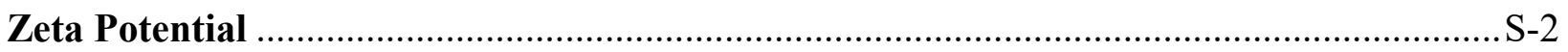

Low-Wavenumber Raman Spectra of Rubrene...................................................... S-3

Extinction and Absorbance Spectra with Scattering Contribution ............................... S-4

Absorbance, Excitation and Emission Spectra …....................................................... S-6

Experimental and Simulated SAED Patterns …................................................... S-10

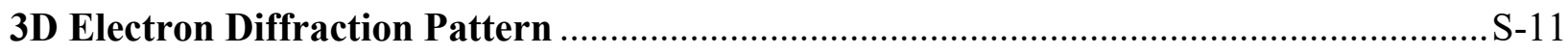

Molecular Packing in Rubrene Polymorphs …..................................................... 12

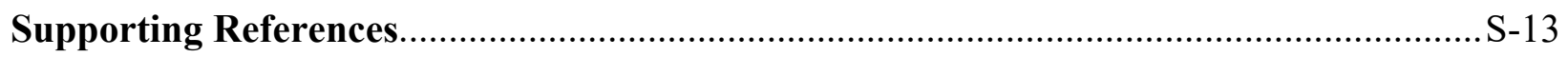




\section{Methods}

Lifetime Measurements. For time-correlated single-photon counting (TCSPC) measurements, the emission from the samples (dispersions, solution, crystals) was directed to a fiber-coupled silicon avalanche photodiode (Micro Photon Devices) and histograms of photon arrival times were built using a counting module (PicoQuant PicoHarp 300). The instrument response function (IRF) was measured for the attenuated laser signal. PL lifetimes were extracted from reconvolution fits using SymPhoTime 64 software.

Zeta Potential Measurements. A Malvern Panalytical Zetasizer Nano ZSP, equipped with a $633 \mathrm{~nm}$ Helium-Neon laser in conjunction with a DTS1070 folded capillary cell was used for zeta potential measurements (zeta analysis v6.1). The capillary cell was equilibrated with the surfactant solution prior to each measurement. The sample viscosity was assumed to be the viscosity of water $\left(1.0031 \mathrm{mPa} \cdot \mathrm{s}\right.$ at $\left.20^{\circ} \mathrm{C}\right)$ and the Smoluchowski approximation was used. Each of the six dispersions was measured five times with a minimum of 10 runs per measurement, with no delay in between measurements. The distribution data of each measurement were fitted using a Gaussian function to extract the center of the Gaussian and averaged. 


\section{Zeta Potential}

Table S1. Average zeta potential values for different fractions of rubrene dispersions in $0.1 \mathrm{~g} / \mathrm{L}$ aqueous sodium cholate solution.

\begin{tabular}{|c|c|c|c|c|c|c|}
\hline & \multicolumn{2}{|c|}{ LPE Orthorhombic Rubrene } & \multicolumn{3}{c|}{ LPE Triclinic Rubrene } \\
\cline { 2 - 6 } & $\begin{array}{c}0-0.1 \\
\mathrm{k} g\end{array}$ & $\begin{array}{c}0.1-1 \\
\mathrm{k} g\end{array}$ & $\begin{array}{c}1-30 \\
\mathrm{~kg}\end{array}$ & $\begin{array}{c}0-0.1 \\
\mathrm{~kg}\end{array}$ & $\begin{array}{c}0.1-1 \\
\mathrm{k} g\end{array}$ & $\begin{array}{c}1-30 \\
\mathrm{k} g\end{array}$ \\
\hline $\begin{array}{c}\text { Zeta } \\
\text { Potential } \\
(\mathbf{m V})\end{array}$ & -30.6 & -21.1 & -15.9 & -22.3 & -26.2 & -19.4 \\
\hline
\end{tabular}




\section{Low-Wavenumber Raman Spectra of Rubrene}

Table S2. Raman shifts of the low-frequency lattice phonons of LPE rubrene and macroscopic single crystals at an excitation wavelength of $785 \mathrm{~nm}$. Light was incident on the $a b$ facet of both orthorhombic as well as triclinic crystals, with the polarization parallel to the short axis of the crystals.

\begin{tabular}{|l|c|c|c|c|c|c|c|c|c|}
\hline & \multicolumn{3}{|c|}{$\begin{array}{c}\text { LPE Orthorhombic } \\
\text { Rubrene }\end{array}$} & \multicolumn{3}{c|}{ LPE Triclinic Rubrene } & $\begin{array}{c}\text { Ortho- } \\
\text { rhombic } \\
\text { Crystal }\end{array}$ & $\begin{array}{c}\text { Triclinic } \\
\text { Crystal }\end{array}$ & $\begin{array}{c}\text { Rubrene } \\
\text { Solution }\end{array}$ \\
\cline { 2 - 8 } & $\begin{array}{c}0-0.1 \\
\mathrm{k} g\end{array}$ & $\begin{array}{c}0.1-1 \\
\mathrm{k} g\end{array}$ & $\begin{array}{c}1-30 \\
\mathrm{k} g\end{array}$ & $\begin{array}{c}0-0.1 \\
\mathrm{k} g\end{array}$ & $\begin{array}{c}0.1-1 \\
\mathrm{k} g\end{array}$ & $\begin{array}{c}1-30 \\
\mathrm{k} g\end{array}$ & & & \\
\hline Low- & 77, & 78, & 77, & 77, & 78, & 77, & 77, & 76, & \\
Wavenumber & 88, & 89, & 87, & 95, & 97, & 97, & 106, & 96, & \\
Raman Shifts & 106, & 107, & 105, & 122, & 123, & 123, & 120, & 122, & \\
$\left.\mathbf{( c m}^{-1}\right)$ & 119, & 120, & 119, & 220 & 219 & 220 & 142, & 219 & \\
& 139, & 140, & 139, & & & & 221 & & \\
& 219 & 221 & 220 & & & & & & \\
\hline
\end{tabular}

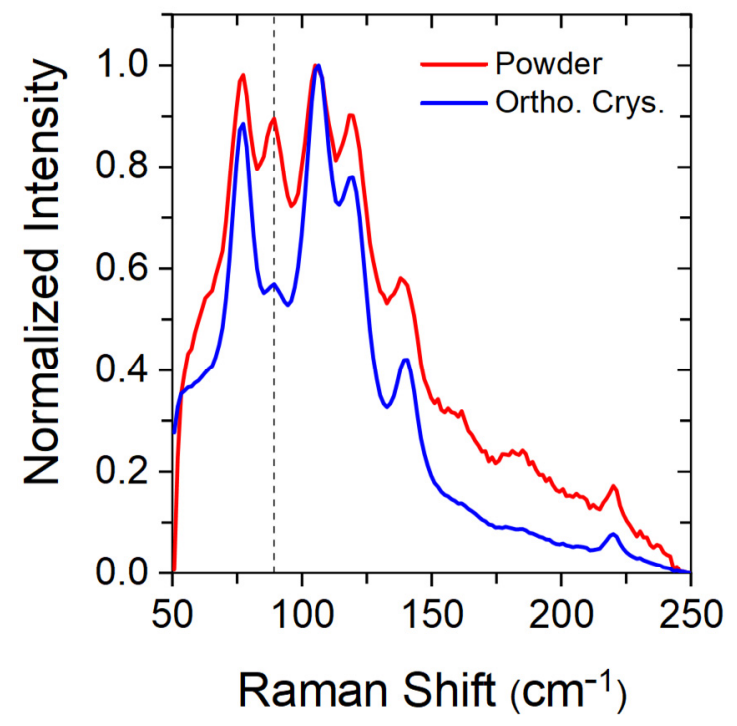

Figure S1. Low-wavenumber Raman spectra (785 nm excitation) of rubrene powder and a macroscopic orthorhombic crystal of rubrene oriented differently under the laser (that is, $a b$-plane not parallel to the substrate). A weak mode at around $88 \mathrm{~cm}^{-1}$ (marked with dashed line) is observed in both cases. 


\section{Extinction and Absorbance Spectra with Scattering Contribution}

Ortho. Rub.

a
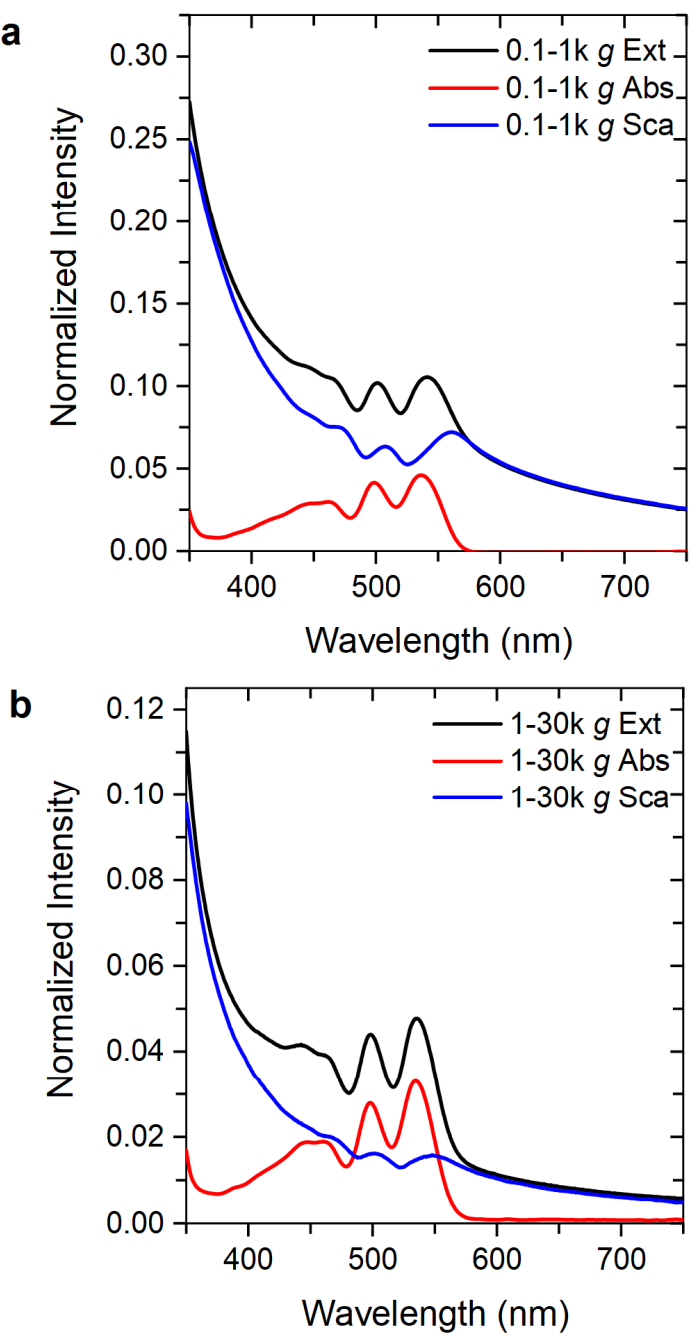

Tri. Rub.
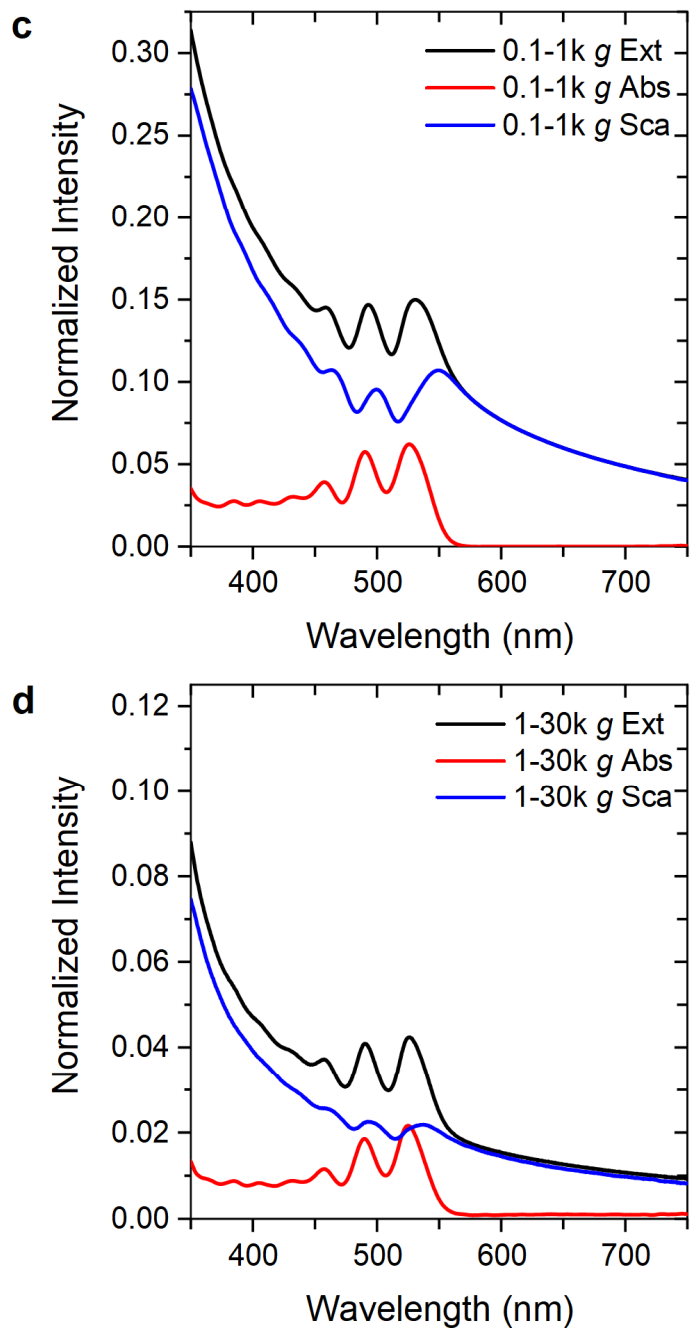

Figure S2. Extinction (Ext) and absorbance (Abs) spectra of the (a) $0.1-1 \mathrm{k} g$ and (b) $1-30 \mathrm{k} g$ fractions of orthorhombic rubrene and the same for triclinic rubrene (c, d). Also shown in each panel are corresponding scattering spectra (Sca) that were obtained by subtracting the absorbance from the extinction spectra. Especially for the $0.1-1 \mathrm{k} g$ fraction, the extinction is dominated by scattering rather than absorbance. In the non-resonant regime (i.e., at energies below the electronic transitions), the scattering follows the characteristic power law. ${ }^{1}$ In the resonant regime, the scattering follows the absorbance in shape, albeit with a red-shift and superimposed on the power law background. Note that this also results in a red-shift of the extinction spectra compared to the absorbance. 


\section{PLE Maps of Exfoliated Rubrene Dispersions and Solution}
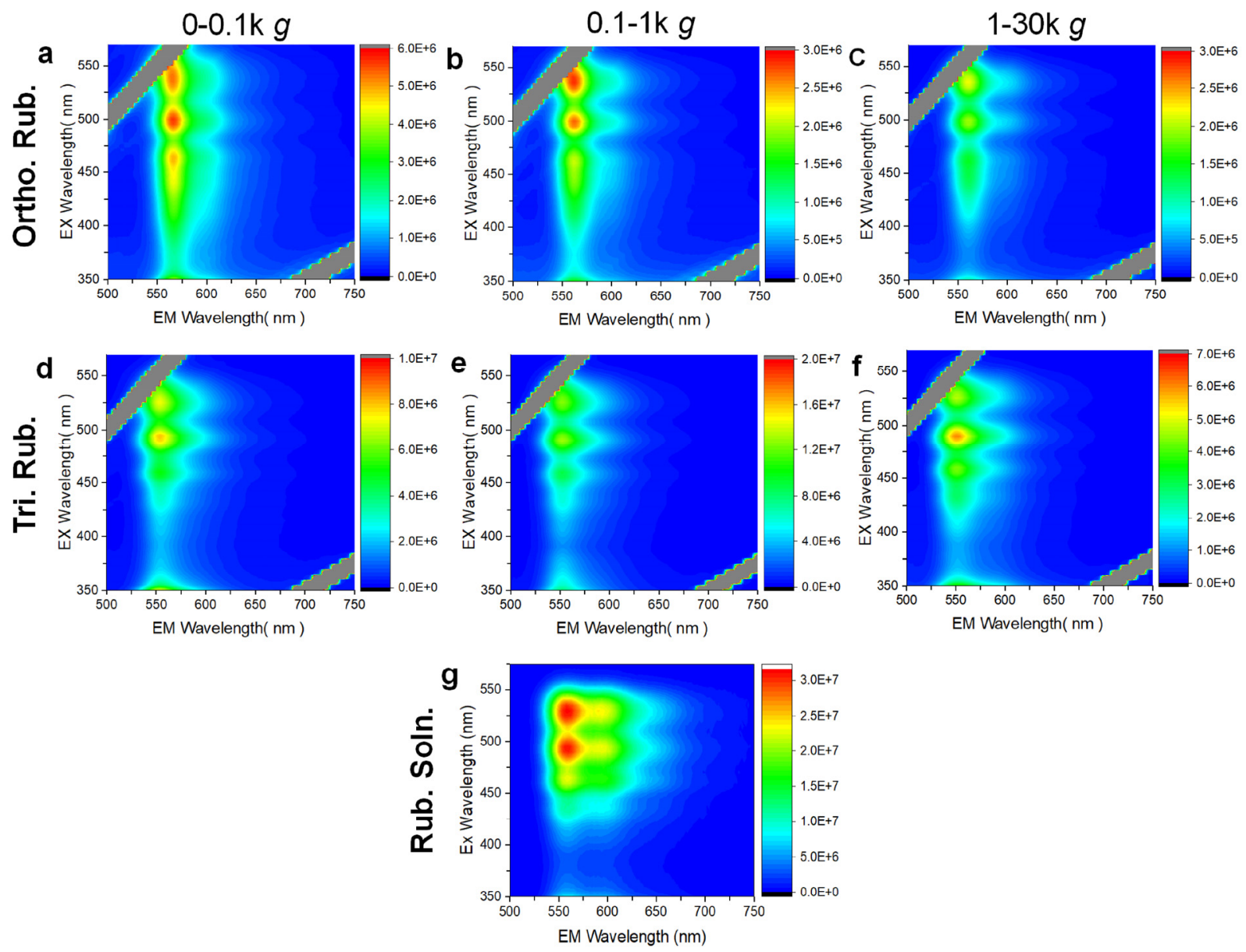

Figure S3. Photoluminescence excitation-emission spectra (PLE maps) of different fractions of liquid phase exfoliated orthorhombic rubrene $(a, b, c)$, triclinic rubrene $(d, e, f)$ dispersions, and a dilute solution of rubrene in chloroform $(\mathrm{g})$. The grey features in the dispersions stem from the excitation. 


\section{Absorbance, Excitation and Emission Spectra}

Rub. Soln.

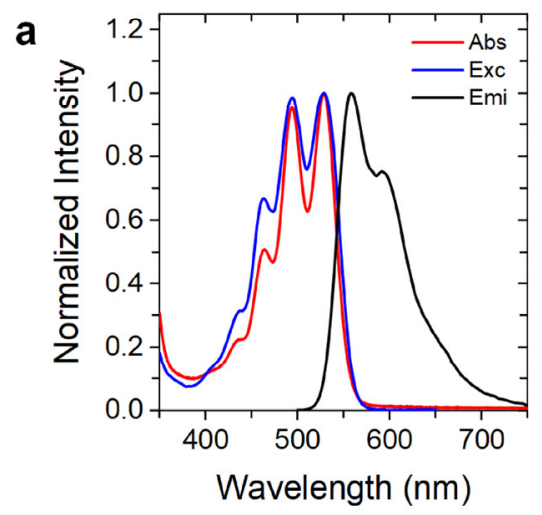

Ortho. Rub.

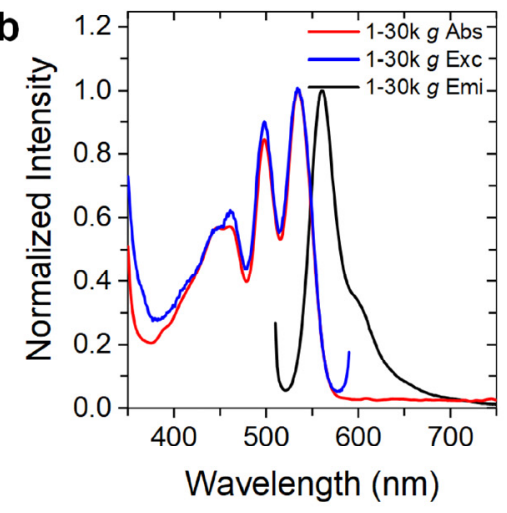

Tri. Rub.

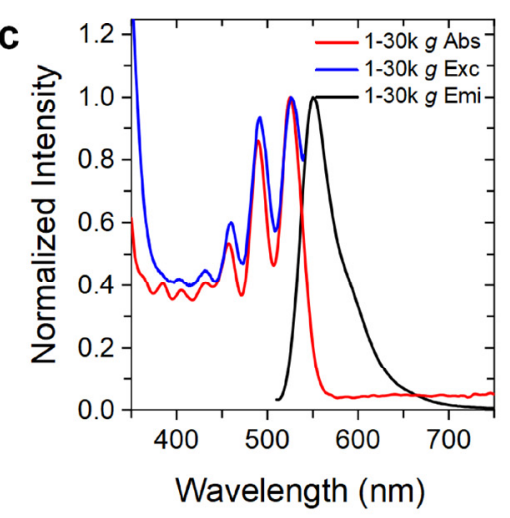

Figure S4. Absorbance (Abs, red), excitation (Exc, blue) and emission (Emi, black) spectra of rubrene solution in chloroform (a), as well as the highest fraction of dispersions of exfoliated othorhombic rubrene (b) and triclinic rubrene (c) in aqueous sodium cholate solution after cascade centrifugation. The excitation spectra show a good overlap with the absorbance spectra. A relatively small Stokes shift is observed in all cases. 
Table S3. Absorbance, Photoluminescnce peak positions of LPE dispersions of orthorhombic and triclinic rubrene and of a solution of rubrene in chloroform.

\begin{tabular}{|c|c|c|c|c|c|c|c|}
\hline & \multicolumn{3}{|c|}{ LPE Orthorhombic Rubrene } & \multicolumn{3}{|c|}{ LPE Triclinic Rubrene } & \multirow{2}{*}{$\begin{array}{l}\text { Rubrene } \\
\text { Solution }\end{array}$} \\
\hline & $\begin{array}{c}0-0.1 \\
\mathrm{k} g\end{array}$ & $\begin{array}{c}0.1-1 \\
\mathrm{k} g\end{array}$ & $\begin{array}{l}1-30 \\
\mathrm{~kg}\end{array}$ & $\begin{array}{c}0-0.1 \\
\mathrm{~kg}\end{array}$ & $\begin{array}{c}0.1-1 \\
\mathrm{k} g\end{array}$ & $\begin{array}{c}1-30 \\
\mathrm{k} g\end{array}$ & \\
\hline $\begin{array}{l}\text { Absorbance } \\
\text { Wavelengths } \\
\text { (nm) }\end{array}$ & $\begin{array}{l}442 \\
463 \\
498 \\
537\end{array}$ & $\begin{array}{l}446 \\
462 \\
498, \\
537\end{array}$ & $\begin{array}{l}447 \\
460 \\
498 \\
535\end{array}$ & $\begin{array}{l}385 \\
406 \\
433 \\
458 \\
490 \\
526\end{array}$ & $\begin{array}{l}385, \\
405, \\
432, \\
458, \\
490, \\
526\end{array}$ & $\begin{array}{l}385, \\
405, \\
432, \\
458, \\
490, \\
525\end{array}$ & $\begin{array}{l}434, \\
464, \\
494, \\
528\end{array}$ \\
\hline \multirow[t]{2}{*}{$\begin{array}{l}\text { PL Position } \\
\text { (nm) }\end{array}$} & $\begin{array}{l}566 \\
604\end{array}$ & $\begin{array}{l}562 \\
604\end{array}$ & $\begin{array}{l}560 \\
604\end{array}$ & 554 & 552 & 550 & $\begin{array}{l}558 \\
592\end{array}$ \\
\hline & \multicolumn{3}{|c|}{$\left(\lambda_{\mathrm{exc}}=500 \mathrm{~nm}\right)$} & \multicolumn{3}{|c|}{$\left(\lambda_{\mathrm{exc}}=495 \mathrm{~nm}\right)$} & $\left(\lambda_{\mathrm{exc}}=495 \mathrm{~nm}\right)$ \\
\hline
\end{tabular}




\section{Photoluminescence Lifetime Measurements}

Ortho. Rub.

a

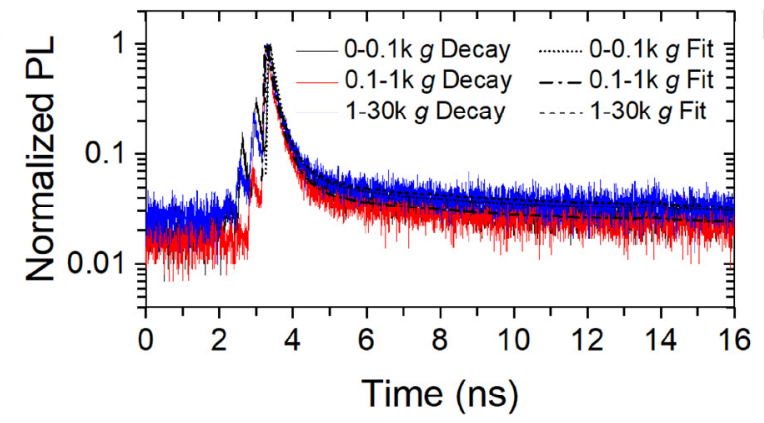

Ortho. Crys.

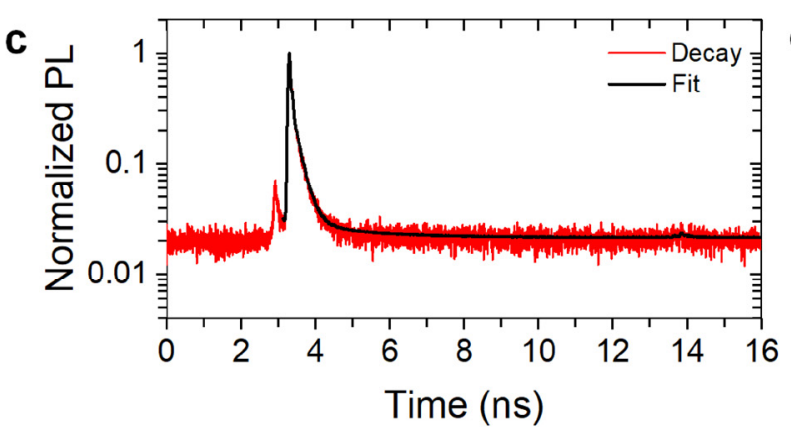

Tri. Rub.

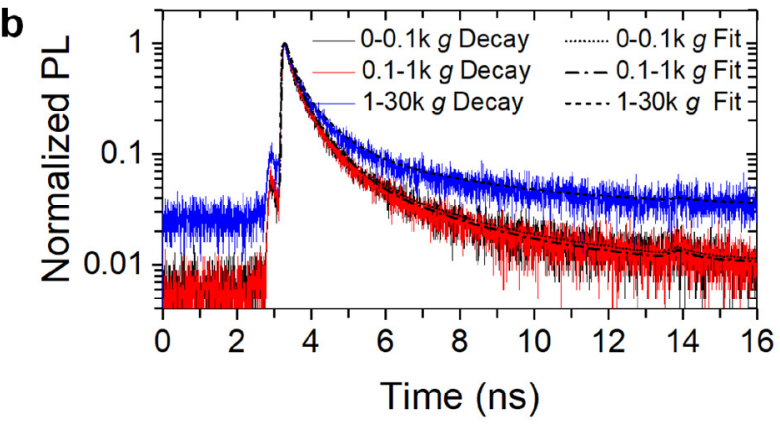

Tri. Crys.

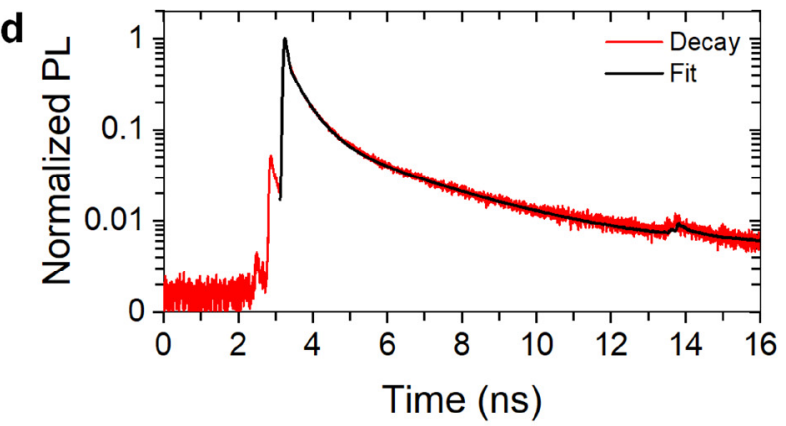

Rub. Soln.

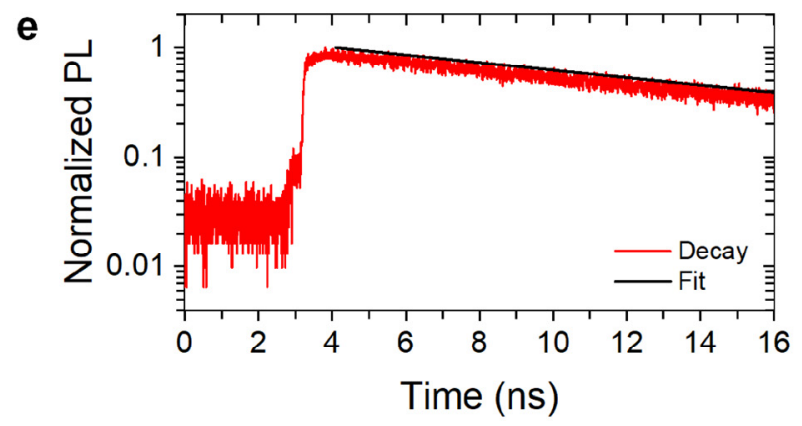

Figure S5. Time-resolved photoluminescence decay traces (time-correlated single-photon counting, TCSPC) of the main emission peak of (a) different fractions of LPE dispersions of orthorhombic crystals, (b) different fractions of LPE dispersions of triclinic crystals, (c) orthorhombic single crystal, (d) triclinic single crystal, and (e) dilute solution of rubrene in chloroform. All samples were excited at $493 \mathrm{~nm}$. Single and multiexponential fits of the PL decay traces are also shown. The extracted lifetimes are provided in Table S4. 
Table S4. Lifetimes (in ns) of different fractions of LPE dispersions of rubrene, orthorhombic and triclinic crystals of rubrene, and solution of rubrene in chloroform.

\begin{tabular}{|c|c|c|c|c|c|c|c|c|c|}
\hline \multirow{2}{*}{$\begin{array}{l}\text { Lifetime } \\
\text { (ns) }\end{array}$} & \multicolumn{3}{|c|}{$\begin{array}{c}\text { LPE Orthorhombic } \\
\text { Rubrene }\end{array}$} & \multicolumn{3}{|c|}{ LPE Triclinic Rubrene } & \multirow{2}{*}{$\begin{array}{l}\text { Ortho- } \\
\text { rhombic } \\
\text { Crystal }\end{array}$} & \multirow[t]{2}{*}{$\begin{array}{c}\text { Triclinic } \\
\text { Crystal }\end{array}$} & \multirow[t]{2}{*}{$\begin{array}{l}\text { Rubrene } \\
\text { Solution }\end{array}$} \\
\hline & $\begin{array}{c}0-0.1 \\
\mathrm{~kg}\end{array}$ & $\begin{array}{c}0.1-1 \\
\mathrm{k} g\end{array}$ & $\begin{array}{c}1-30 \\
\mathrm{~kg}\end{array}$ & $\begin{array}{c}0-0.1 \\
\mathrm{~kg}\end{array}$ & $\begin{array}{c}0.1-1 \\
\mathrm{k} g\end{array}$ & $\begin{array}{l}1-30 \\
\mathrm{~kg}\end{array}$ & & & \\
\hline$\tau_{1}$ & 0.08 & 0.08 & 0.05 & 0.11 & 0.11 & 0.13 & 0.01 & 0.03 & \\
\hline$\tau_{2}$ & 0.56 & 0.44 & 0.52 & 0.70 & 0.68 & 0.72 & 0.19 & 0.50 & $\tau=12$ \\
\hline$\tau_{3}$ & 3.90 & 4.10 & 5.70 & 3.10 & 2.80 & 3.40 & 1.70 & 2.80 & \\
\hline
\end{tabular}




\section{Experimental and Simulated SAED Patterns}

a

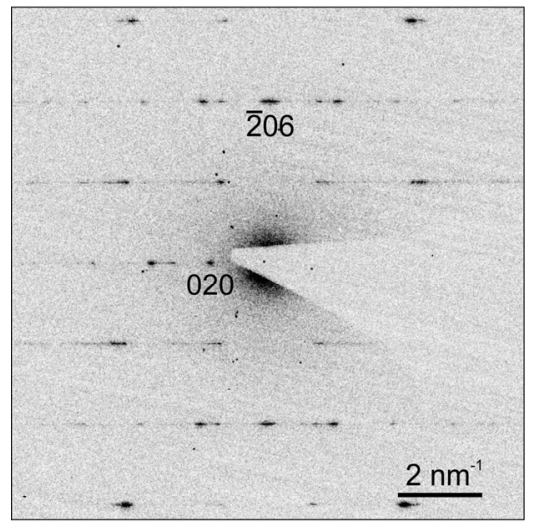

d

$[\overline{3} 0 \overline{1}]$

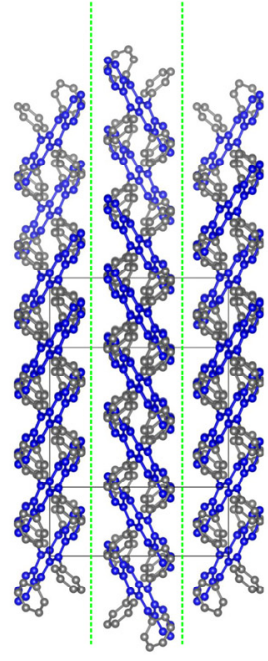

(020) b

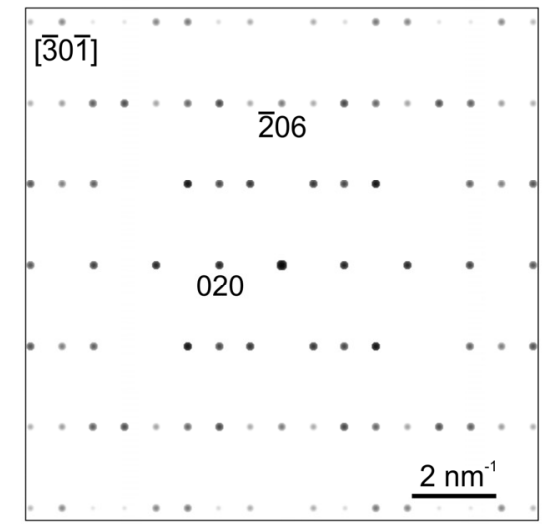

e

$[00 \overline{1}]$
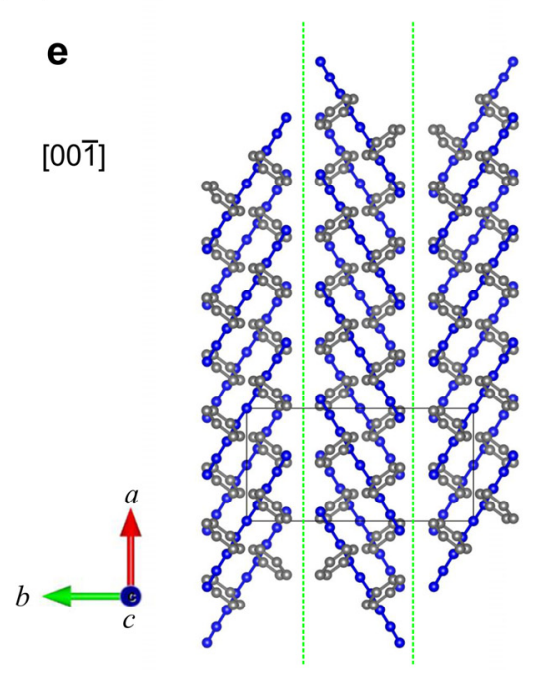

(020)

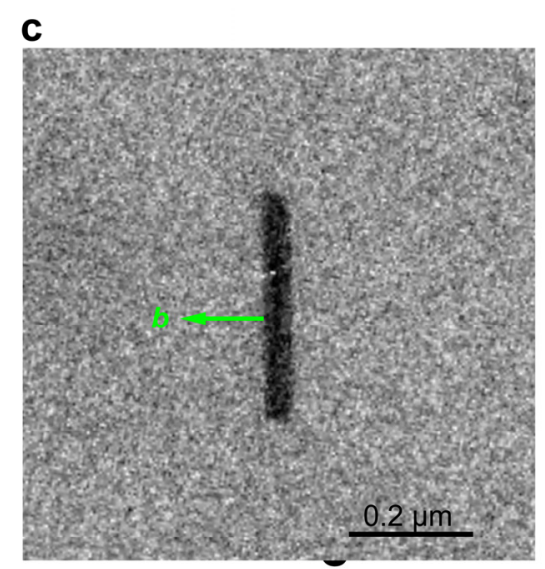

f

[0̄̄o]

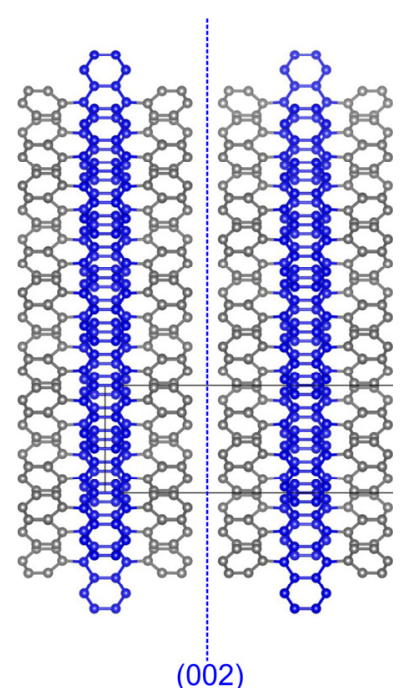

Figure S6. (a) Experimental SAED pattern of the nanorod. The nearest reflections along the high symmetry axes are at $1.4 \mathrm{~nm}^{-1}$ and $3.7 \mathrm{~nm}^{-1}$, corresponding to the 020 and 206 reflections of orthorhombic rubrene, respectively. (b) Simulated SAED pattern of orthorhombic rubrene along the $[\overline{3} 0 \overline{1}]$ zone axis. The simulated diffraction pattern agrees well with the experimental pattern in (a). And the simulated 020 and 206 reflections are at $1.4 \mathrm{~nm}^{-1}$ and $3.6 \mathrm{~nm}^{-1}$, respectively. (c) Bright-field TEM image of the nanorod, from which the experimental SAED pattern in (a) was acquired. The image and the SAED pattern were rotated by the same degrees to facilitate discussion. By comparing (c) with (a), we found that the short edge of the nanorod is parallel to the [020] direction. Thus the $b$-axis (i.e., [010] vector) is perpendicular to the long axis of the nanorod (as depicted by the green arrow). (d) Atomic model of orthorhombic rubrene viewed in $[\overline{3} 0 \overline{1}]$ projection. The (020) plane (i.e., ac-plane, marked by green dashed lines) is parallel to the long edge of the nanorod in (c), suggesting that the delamination occurred primarily at the $a c$ plane. (e) Atomic model of orthorhombic rubrene viewed in [001] projection, showing the herringbone configuration and thus weaker intermolecular interaction across the $a c$-plane. Along the $a$-axis, the molecules are packed via $\pi-\pi$ interaction. (f) Atomic model of orthorhombic rubrene viewed in [0 $\overline{1} 0]$ projection. The blue dashed line denotes the (002) plane (i.e., ab-plane). Since there is no strong intermolecular force across the $a b$-plane, delamination is also highly plausible. 


\section{D Electron Diffraction Pattern}
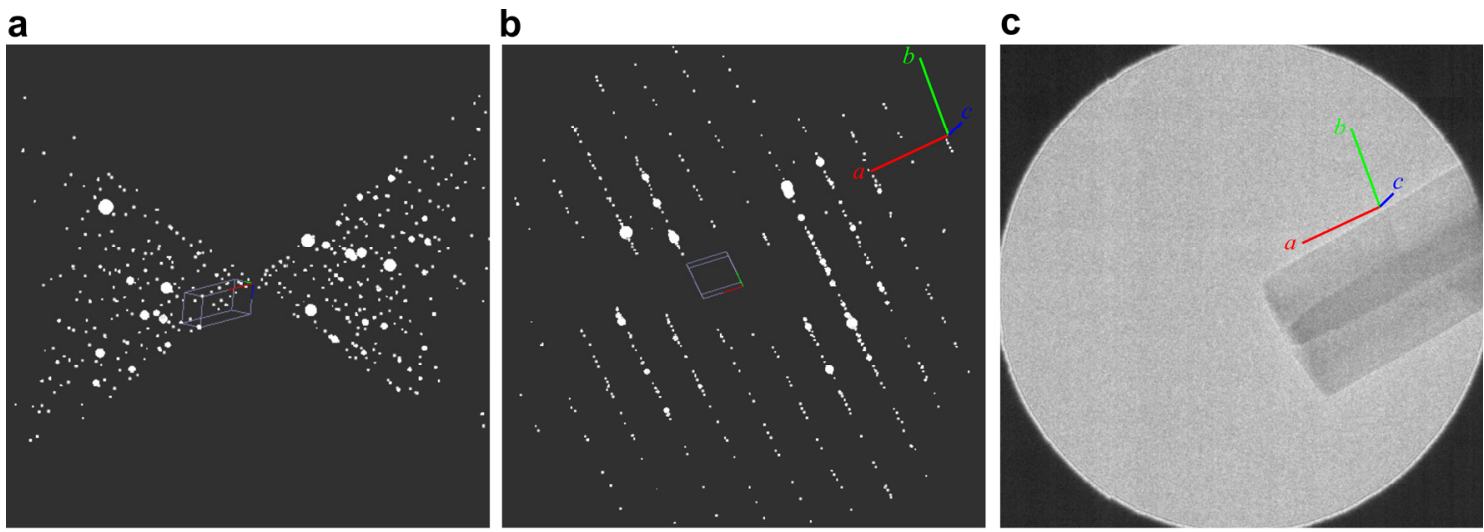

d

\section{e}
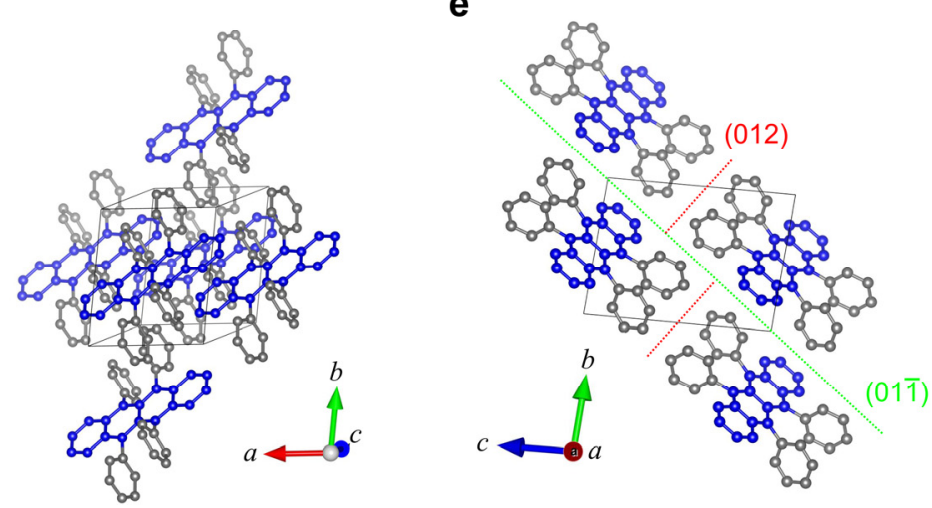

Figure S7. (a) Reconstructed 3D electron diffraction pattern of the triclinic unit cell of rubrene via continuous rotation electron diffraction (cRED). The solid lines represent the reciprocal lattice vectors: $a^{*}$ (red), $b^{*}$ (green), and $c^{*}$ (blue). The unit cell parameters have been determined as: $a=7.04 \AA, b=8.57 \AA, c=12.21 \AA, \alpha=93.9^{\circ}, \beta=105.8^{\circ}, \gamma=95.4^{\circ}$, in agreement with the triclinic rubrene $\left(a=7.02 \AA, b=8.54 \AA, c=11.95 \AA, \alpha=93.0^{\circ}, \beta=105.6^{\circ}, \gamma=96.3^{\circ}\right)$. (b) Projection of the $3 \mathrm{D}$ electron diffraction pattern at the tilt angle of $0^{\circ}$. The reciprocal unit cell is shown in the center. The real lattice vectors are presented in the upper right corner. (c) TEM image of the triclinic rubrene needle at the tilt angle of $0^{\circ}$ with the real lattice vectors overlaid. The projection of the $a$-axis is almost parallel to the long edge of the needle crystal. (d) Atomic structure of the triclinic rubrene. The view direction is identical as in (b) and (c), with the projected $a$-axis aligned horizontally. When rotating this model about the vertical axis by $90^{\circ}$, the view direction is exactly parallel to the $a$-axis, as shown in (e). In other words, the $a$ direction is along the long axis of the needle crystal in (b). The dashed lines illustrate the possible cleavage planes, i.e., (01 $\overline{1})$ and (012), due to weaker intermolecular interactions. 


\section{Molecular Packing in Rubrene Polymorphs}

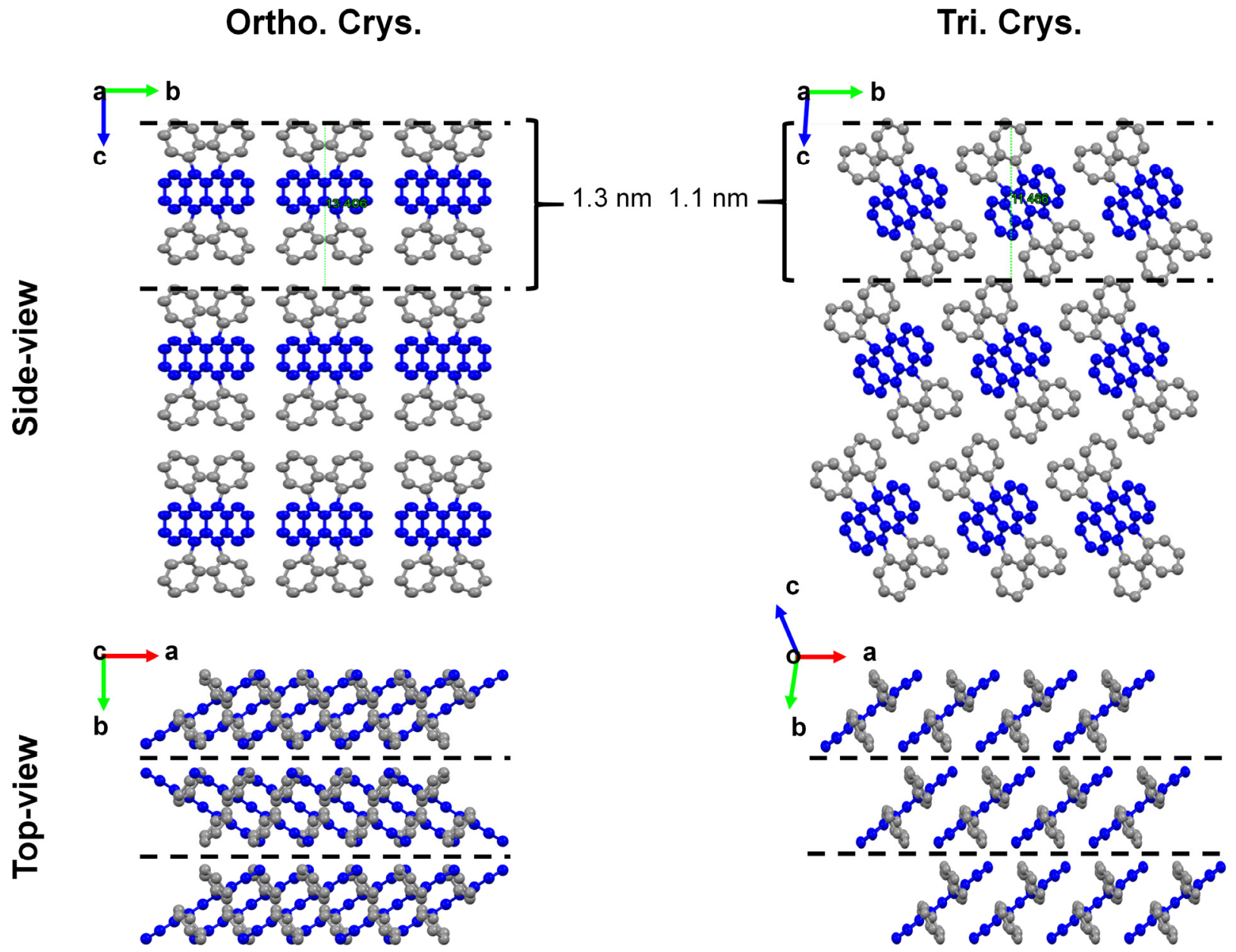

Figure S8. Top and side views of the arrangement of rubrene molecules in the orthorhombic (Ortho.) as well as the triclinic (Tri.) crystals. The interplanar distances of $1.3 \mathrm{~nm}$ and $1.1 \mathrm{~nm}$ in orthorhombic and triclinic lattices, respectively, are indicated in the side-view of both lattices. The direction of the strongest $\pi-\pi$ interactions (along $a$-axis) and the most probable set of planes of exfoliation: (020) for the orthorhombic crystal and (011) for the triclinic crystal, are shown in the top-view images. 


\section{Supporting References}

1. Harvey, A.; Backes, C.; Boland, J. B.; He, X.; Griffin, A.; Szydlowska, B.; Gabbett, C.;

Donegan, J. F.; Coleman, J. N., Non-Resonant Light Scattering in Dispersions of 2D Nanosheets.

Nature Commun. 2018, 9, 4553. 